\title{
Aprendizaje-Servicio, docencia e implicación social del profesorado universitario. ¿Existe alguna relación?
}

\author{
Service-Learning, teaching and social involvement of university teachers. Is there any \\ connection?
}

\author{
Alexandre Sotelino-Losada*, Igor Mella-Núñez **, Cristina Varela-Portela ** \\ * Universidade de Vigo - GI-Esculca (USC); ** GI Esculca - Universidade de Santiago de Compostela
}

\begin{abstract}
Resumen
El objetivo de esta comunicación será evaluar en qué medida la participación social del profesorado universitario en entidades del tercer sector ejercerá una influencia en su docencia, y también, en su opinión al respecto del aprendizaje-servicio. Para ello hemos realizado un estudio con metodología ex-post-facto (no experimental), con método descriptivo de encuesta. La población elegida ha sido el profesorado de la Universidad de Santiago de Compostela (muestra de 326 sujetos). Concluimos que la implicación en proyectos vinculados a estas entidades, parece que se relaciona con una mirada de la docencia más allá de las aulas, así como con entender la responsabilidad social de la Universidad como un factor clave.

Palabras clave: Aprendizaje-servicio, participación del profesor, transferencia, universidad, enseñanza superior
\end{abstract}

\section{Abstract}

The aim of this paper is to evaluate the extent to social participation of university teachers in the third sector will exert an influence on their teaching, and also, in their opinion on service-learning. So we've made a study of ex-post-facto (not experimental), with descriptive method of survey methodology. The population has been the faculty of the University of Santiago de Compostela (Sample of 326 subjects). We conclude that involvement in projects related to these entities, it seems that relates to a look of teaching beyond the classroom and understand the social responsibility of the University as a key factor.

Keywords: Service-learning, teacher participation, transference, university, higher education

\section{Introducción}

En nuestro marco de referencia político, la Universidad tiene sus propias formas institucionales de relacionarse con el ambiente social. Los Consejos sociales son una vía, ya que, como órganos colegiados, sirven como un encuentro entre sociedad civil y academia, facilitando la comunicación y colaboración entre los dos organismos para ayudar al desarrollo educativo, social, económico, cultural, laboral y tecnológico. Nacidos a partir de la Ley 6/2001, del 21 de diciembre, de Universidades (BOE 24/12/2001), modificada por la Ley Orgánica 4/2007 de 12 de abril de 2007 (BOE 13/04/2007) son definidos como: “(...) el órgano de participación de la sociedad en la Universidad". En Galicia, las funciones de los Consejos Sociales están especificadas en la Ley 1/2003, de 9 mayo, de los Consejos Sociales del Sistema
Universitario de Galicia (DOG 30/05/2003), donde la definición se explicita afirmándose que se trata del “(...) órgano de participación de la sociedad gallega en las universidades públicas que integran el Sistema Universitario de Galicia, que impulsa la colaboración entre estas y aquella mediante la satisfacción por las universidades de las necesidades de su contorno, contribuyendo eficazmente al desarrollo social, profesional, económico, tecnológico y cultural de Galicia, al avance de la calidad del servicio público de la educación superior universitaria y más a la obtención de los recursos precisos para procurar la su suficiencia económica y financiera". En el trabajo que ha editado la Universidad de Santiago de Compostela con motivo de la celebración del 25 aniversario de su Consejo Social, se comenta que estos organismos tienen un importante potencial ante los retos del nuevo sistema de enseñanza superior, en tanto que mantenimiento de su prestigio dentro y fuera de la Universidad y en la adaptación de sus competencias (Consello Social, 2013).

Pero algo que debemos preguntarnos, como punto de partida de este trabajo, es si son suficientes estos canales de relación universidad-sociedad, o por el contrario, deberíamos hacer lo posible por optimizar tales vínculos. La evolución de la práctica universitaria nos sugiere que tal vez debiéramos cuestionar esta visión y valorar su replanteamiento, por ejemplo en lo tocante a la docencia y la relación teoría-práctica que implica.

Aprendizaje y participación social en la Universidad no se pueden definir como un binomio en el proceso educativo ya que, tradicionalmente, el rol de alumnos y profesores ha tenido poca influencia en el entorno social circundante. En este sentido, se ha visto como la Academia ha ido tomando conciencia de la importancia que tiene la implicación social de la comunidad universitaria, tanto para su visualización como para la optimización de los aprendizajes. Este proceso se ha agudizado a partir de final de los años 90, una vez reestructuradas las universidades europeas, debido a la creación del Espacio Europeo de Educación Superior, al que nos referiremos posteriormente en mayor medida.

Tiene también especial importancia en el ámbito español la promulgación de la Ley estatal del Voluntariado (Ley 6/1996, de 15 de enero, del Voluntariado) a partir de la cual las universidades españolas comienzan a incorporar a su oferta de 
extensión universitaria servicios y/o programas que favorecían la participación del alumnado en proyectos de su entorno.

Como comentábamos, aún es reciente la participación del alumnado en diferentes espacios universitarios. Inicialmente esta participación se ha dado al margen de las actividades académicas, principalmente en actividades culturales o deportivas ligadas a la institución. Desde hace ya varios años, las universidades del Estado comenzaron a integrar dentro de sus estructuras y de forma más o menos estable, servicios o programas que tienden a favorecer la participación de la comunidad universitaria dentro de las actividades y propuestas desarrolladas por las entidades de acción social existentes en los entornos más próximos de sus campus. Estos son los servicios de voluntariado, extensión universitaria o participación, entre otras muchas denominaciones que han recibido (Arias \& Simón, 2004). A continuación presentamos una figura que ofrece una panorámica histórica de los cambios que han ido aconteciendo en las universidades y como han afectado a sus actividades sociales.

\begin{tabular}{|c|c|c|}
\hline $\begin{array}{l}\text { Acceso elitista } \\
\text { a la Educación } \\
\text { Superior }\end{array}$ & & $\begin{array}{r}\text { Masificación } \\
\text { del Acceso a la } \\
\text { Educación Superior }\end{array}$ \\
\hline $\begin{array}{l}\text { Universidad vista como } \\
\text { Torre de Marfil }\end{array}$ & $\begin{array}{l}\text { Universidad } \\
\text { Abierta }\end{array}$ & $\begin{array}{r}\text { Universidad } \\
\text { Socialmente } \\
\text { Responsable }\end{array}$ \\
\hline $\begin{array}{l}\text { - Formación Prolesional } \\
\text { - Investigación básica }\end{array}$ & $\begin{array}{l}\text { - Investigación } \\
\text { aplicada } \\
\text { - Transferencia del } \\
\text { conocimiento } \\
\text { - I+D D i } \\
\text { - Creación de spin-off }\end{array}$ & $\begin{array}{r}\text { - Aprendizaje servicio } \\
\text { - Educación para toda } \\
\text { la vida } \\
\text { - Cooperación al } \\
\text { desarrollo } \\
\text { - Voluntariado } \\
\text { universitario } \\
\text { - Liderazgo social } \\
\text { - Huella ecológica } \\
\text { (sustentabilidad) }\end{array}$ \\
\hline
\end{tabular}

Figura 1: Cambios en la Responsabilidad Social Universitaria (Gaete, 2011, p. 130).

Este proceso no ha sido por motivos azarosos sino que el propio contexto internacional parecía caminar con sensibilidad para atender la integridad educativa de la persona, en todos los niveles educativos y por supuesto también en el ámbito universitario; Kymlicka (2003) define este fenómeno como "ascenso del civismo". Para Bowen (1984) los departamentos, facultades o escuelas universitarias, teniendo como referencia la responsabilidad social, asumen dos grandes compromisos: uno técnico y otro moral. La responsabilidad técnica, en tanto que proporciona profesionales técnicamente competentes a la sociedad, bien entrenados, autodisciplinados y con un claro interés por seguir aumentando su aprendizaje a lo largo de su vida. Y por otro lado, la responsabilidad moral, aludiendo a la formación de personas con conocimientos y cultura extensa, con capacidad para asumir liderazgo en la sociedad, amén de ejercer una influencia constructiva en la comunidad y en la vida cívica (Santos Rego \& Lorenzo, 2007). Por lo tanto, la educación superior debe preparar a sus alumnos de tal manera que lleguen a ser ciudadanos, reflexivos, críticos, capaces de pensar y reflexionar autónomamente (Ugarte \& Naval, 2010). En otras palabras, las instituciones educativas han de preparar para la participación e implicación social. Ya en los años 70, Otero (1974, p. 110) abogaba por un educando participante, considerando que la "toma decisiones implica problemas de motivación y actitud y problemas de conocimiento". El hecho de tener que discernir entre varias alternativas de procedimiento, y saber las consecuencias que puede traer su decisión, implica un mayor conocimiento de las variables; y, por consiguiente, la necesidad de acceder a la información.

\section{Aprendizaje-servicio y participación en la Universidad}

En su último libro sobre el tópico, Josep María Puig (2009, p. 9) define el aprendizaje-servicio (ApS) como "una metodología que combina en una sola actividad el aprendizaje de contenidos, competencias y valores con la realización de tareas de servicio a la comunidad. En el aprendizaje-servicio el conocimiento se utiliza para mejorar algo de la comunidad y el servicio se convierte en una experiencia de aprendizaje que proporciona conocimientos y valores". Hay que destacar el hecho de esta definición cite el ApS como "propuesta pedagógica" y no como "metodología" lo que modifica sustancialmente la concepción.

En nuestro ámbito más inmediato, refiriéndonos a la Comunidad Gallega, son los profesores Miguel A. Santos Rego y Mar Lorenzo Moledo los que más se han hecho eco de esta metodología. En una de sus publicaciones hacen referencia al aprendizaje-servicio como " $(\ldots)$ actividades escolares con clara proxección social. O obxectivo é que os alumnos adquiran os contidos académicos programados dende o currículo das diferentes materias, pero debe facerse realizando un servizo de utilidade social no medio" (Lorenzo \& Santos Rego, 2009, p. 81). Los mismos Profesores de la Universidad de Santiago de Compostela observan en otra de sus recientes obras que en Galicia, la eficacia de esta metodología de aprendizaje-servicio se podría ver reforzada por la gran cantidad de estímulos ligados al territorio y a la cultura, elementos intrínsecos de una realidad histórica en la que una propuesta de estas características supone oportunidades para el desarrollo del aprendizaje y de la comunidad (Santos Rego, Sotelino \& Lorenzo, 2015).

En definitiva, el ApS supone ir un paso más allá de otros tipos de metodologías de servicio a la comunidad. El aprendizaje-servicio se diferencia por ofrecer al mismo tiempo una alta calidad de servicio en relación con un alto nivel de aprendizaje (Santos Rego, 2013). Esto se consigue mediante una vinculación explícita con el currículum académico, en el caso de la educación reglada, o con el proyecto educativo como referente para otro tipo de instituciones. En este sentido, el hecho de que el profesorado participe activamente en entidades del tercer sector, debería facilitar una relación pragmática entre curriculum y realidad social, puesto que el docente se sitúa en un puesto de interlocutor, conociendo de primera mano ambas instancias. 


\section{Aprendizaje-servicio y pertinencia en la Educación Superior}

La Universidad constituye un contexto propicio para la educación cívica, aunque parece que concentra todos sus esfuerzos en la calificación técnica de sus alumnos, sin considerar suficientemente otras variables en el marco de las competencias cívico-sociales, aspecto clave en la formación universitaria y en la convergencia europea. Ugarte y Naval (2010) nos hablan de dos tipos de competencias que el alumnado universitario debería desarrollar:

- Competencias intelectuales: las que facilitan la conquista del pensamiento crítico, promoviendo que sean ciudadanos capaces de hacer una crítica abierta y al mismo tiempo constructiva de las realidades que les rodean. Se destacan, entre otras, el liderazgo personal, la integridad y la capacidad de tomar decisiones.

- Competencias participativas: las que ayudan a fomentar y reforzar el compromiso cívico, ejerciendo una ciudadanía responsable. Entre estas destacan las habilidades de comunicación, negociación, resolución de problemas, iniciativa personal y el trabajo en equipo.

Creemos, por lo tanto, que se debe seguir articulando una pedagogía de la sociedad civil que aproveche la oportunidad de sensibilizar a alumnos, profesores y demás personal a favor de una tarea que no solo redundará en beneficios personales sino también profesionales. Lo que se resumiría en aprender de la vida y aprender para la vida. Siguiendo a Martínez (2008, p. 23) “(...) dado que el mundo de los valores se aprende en la cotidianidad de aprender y del convivir, la Universidad no puede ser un espacio externo al aprender y al convivir $\mathrm{y}$ es un buen lugar para aprender éticamente. Obviamente, no todas las formas de enseñar y aprender son adecuadas para (...) construir matrices de valores personales orientadas a la consolidación de estilos de vida personal y comunitaria, activa y democrática, de igual forma ocurre con las propuestas de aprendizaje-servicio". Esto se entiende como abrir las puertas de la Universidad al conocimiento, para que este pueda salir y entrar en relación con el entorno. Tenemos la convicción de que la universidad moderna tiene que convertirse en un instrumento clave de la sociedad del siglo XXI, para “(...) la formulación y transferencia de valores sociales establecidos y legítimos, para el desarrollo de la próxima generación de ciudadanos y para la socialización política" (García, Escámez, Martínez \& Martínez, 2008, p. 83).

Puede quedar claro, entonces, que la Universidad tiene una responsabilidad social, más allá del cauce institucional de los Consejos Sociales o las otras vías que anteriormente señalábamos. Lo que se tendrá que concretar en propuestas pragmáticas con el alumnado por medio de los planes de estudio, procurando vínculos con la comunidad desde la que se puede aportar un ingrediente humano a los currículos demasiado formalizados. En los tiempos que corren será bueno que el profesorado asuma de manera clara estas premisas y busque nuevas formas de orientar sus materias para que, además de aprender contenidos específicos, se pueden transmitir valores cívico-sociales (Lorenzo, 2012).
Cuando proponemos el aprendizaje-servicio como metodología es porque favorece el establecimiento de un vínculo real entre el currículum y las realidades sociales inmediatas. En consecuencia, el aprendizaje no solo se haría más contextualizado, sino también más conectado con las dimensiones que pueden informar más tarde el desarrollo de su actividad profesional.

\section{Método}

El objetivo central de este trabajo pasa por analizar en qué medida la implicación del profesorado en entidades del tercer sector tendrá influencia en su forma de ejercer la docencia y sobre el conocimiento del aprendizaje-servicio.

Para alcanzar este objetivo hemos optado por realizar una investigación cuantitativa. Al ser el primer estudio de este tipo en Galicia, debíamos evaluar la situación real de la que se partía para, en segundo lugar, delimitar el estado de la cuestión (Del Rincón, Arnal, Latorre \& Sans, 1995). A tal efecto, nuestro trabajo ha seguido una metodología ex-post-facto (no experimental), con un método descriptivo de encuesta entre profesorado universitario (Sotelino, 2014).

La población elegida de estudio ha sido el profesorado de la Universidad de Santiago de Compostela, en sus tres Campus (dos en Santiago de Compostela y uno en Lugo). La selección de la muestra (326 sujetos) se ha realizado mediante muestreo estratificado por áreas de conocimiento procurando la representatividad de la muestra (Ciencias de la Salud, Ciencias Sociales y Jurídicas, Ciencias Experimentales, Enseñanzas Técnicas y Humanidades). Los estratos tienen una afijación proporcional en función del número de elementos que componen cada uno, así es que el estrato mayor es el de Ciencias Sociales y Jurídicas, puesto que la mayoría de las titulaciones de la Universidad de Santiago de Compostela pertenecen a este grupo $(35 \%$ del total).

Finalmente, la muestra productora de datos ha estado constituida por: 60 profesores de Ciencias de la Salud, 52 de Ciencias Experimentales, 132 de Ciencias Sociales y Jurídicas, 33 de Enseñanzas técnicas y 47 de Arte y Humanidades.

Como instrumento elegimos el más utilizado en la investigación por encuesta, esto es, un cuestionario diseñado específicamente para el estudio. Tras realizar una extensa revisión bibliográfica sobre el tema, elaboramos una batería de 45 ítems, que se estructuran en diferentes bloques temáticos. Cabe destacar que el cuestionario ha alcanzado una fiabilidad que supera el 0,70 en todos los bloques, por lo que se considera aceptable según el índice alfa de Cronbach.

Para este trabajo utilizaremos como variable independiente la participación del profesorado en entidades del tercer sector, y las variables dependientes serán las actitudes, preferencias y conocimientos del profesorado universitario sobre su docencia en general y, de manera más concreta, en relación al conocimiento y pertinencia del aprendizaje-servicio. 


\section{Resultados}

Respecto a la participación de los docentes en el movimiento asociativo cívico-social, los datos muestran que la mitad del profesorado de la muestra forma parte de alguna entidad (Véase Gráfico 1). Esto indica que existe algún compromiso social con el entorno, aunque también debemos advertir que, en muchas ocasiones, esta pertenencia a colectivos se limita al simple pago de las cuotas de socios pero no a una participación activa. De todas formas, nos parece un dato alentador pues puede favorecer el desarrollo del aprendizaje-servicio, teniendo en cuenta la importancia de las entidades del entorno social a la hora de impulsar la metodología que centra nuestro estudio.

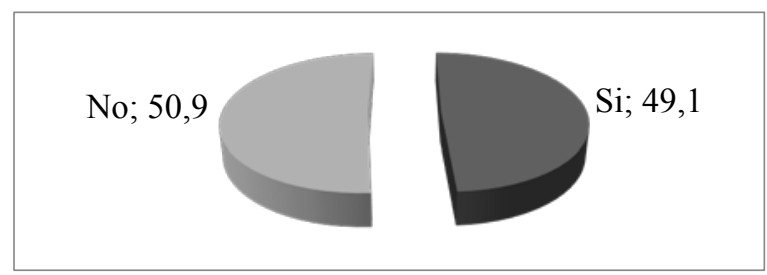

Grafico 1. Pertenencia a alguna asociación, ONG, fundación o entidad cívico-social

Entendemos que la pertenencia y/o implicación en una entidad de corte cívico-social es un reflejo del compromiso social de la persona. Lo que nos interesa saber a continuación es si las respuestas de los sujetos respecto de su docencia y el conocimiento del aprendizaje-servicio varían en función de esta circunstancia.

En el primer bloque relativo a la docencia, podemos observar que en la mitad de los ítems encontramos diferencias significativas. Para hacer más comprensible este estudio vayamos analizándolas por partes.

Respecto de los ítems cuyo eje central gira en torno a la metodología de aula, los encuestados que pertenecen a alguna entidad $(\overline{\mathrm{x}} 1=2,48)$ invitan más a profesionales externos a la Universidad para exponer su trabajo, que sus compañeros $(\overline{\mathrm{X}} 2=2,08)$, afirmando que estas diferencias son estadísticamente significativas $(t=3,234$; $\mathrm{p} \leq 0,001)$. También son estos profesores implicados los que más afirman $(\overline{\mathrm{x}} 1=3,76)$ la participación activa de los alumnos en clase, mientras que los que no pertenecen a ningún movimiento lo hacen en menor medida $(\bar{x} 2=$ $3,52)$. De nuevo, cabe afirmar que existen diferencias significativas $(\mathrm{t}=4,245 ; \mathrm{p} \leq 0,021)$. Por otro lado, los grupos utilizan bastante los recursos tecnológicos en el aula.

En segundo lugar, nos centramos en aquellos enunciados que hacen referencia a actividades desarrolladas fuera del aula. Los encuestados que participan del movimiento social recomiendan más a sus alumnos visitar exposiciones o acudir a actos relacionados con la materia $(\overline{\mathrm{X}} 1=3,37)$, que los que no pertenecen a ninguna entidad cívica $(\overline{\mathrm{X}} 2=3,04)$. En todo caso, la diferencia de medias entre los grupos es estadísticamente significativa $(\mathrm{t}=2,497 ; \mathrm{p} \leq 0,013)$.

Se comprueban también diferencias $(\mathrm{t}=2,631$; $\mathrm{p} \leq 0,014$ ) entre los profesores a la hora de organizar actividades complementarias al margen del horario lectivo. Los asociados alcanzan una media de 2,42, y el otro grupo tiene una media inferior $(\bar{x} 2=2,09)$. Esta tendencia cambia al relacionar su materia con el practicum de la titulación, ya que en este caso son los docentes que no pertenecen a asociaciones $\mathrm{u}$ otras entidades cívico-sociales los que más lo hacen $(\overline{\mathrm{x}} 2=$ 3,23).

Ya en la evaluación encontramos diferencias en las dos variables. Los sujetos que pertenecen a entidades cívicas realizan con frecuencia evaluación continua $(\bar{x} 1=4,08)$, mientras que los que no militan en ningún movimiento de esta índole no alcanzan esta puntuación $(\bar{x} 2=3,76)$. Estas diferencias son estadísticamente significativas, ya que la prueba $t$ de Student, indica que para un valor $\mathrm{t}=2,654 ; \mathrm{p} \leq 0,008$. Son estos mismos docentes los que también consideran la participación e implicación del alumnado en su evaluación $(\bar{X} 1=4,24)$ marcando diferencias significativas $(t=2,70 ; p \leq 0,014)$ con el otro grupo $(\overline{\mathrm{X}} 2=4,01)$. En el último apartado nos centraremos en ítems sobre actividades para la toma de decisiones. El colectivo de docentes que pertenecen a organizaciones cívico-sociales son más proclives $(\overline{\mathrm{X}} 1=4,04)$ a promover actividades que fomenten el pensamiento crítico que los que no tienen ese compromiso social $(\bar{x} 2=3,68)$. La diferencia de medias es, en todo caso, significativa $(\mathrm{t}=3,658 ; \mathrm{p} \leq 0,000)$. Sin embargo, no hay diferencias significativas entre los dos grupos a la hora de fomentar actividades que promuevan la toma de decisiones técnicas.

También se ha preguntado a los sujetos de la muestra sobre su conocimiento del aprendizaje-servicio y su pertinencia en la Universidad (Véase Gráfico 2). Estas cuestiones eran dicotómicas (Sí/No), con un apartado de carácter abierto solo en los casos afirmativos de conocimiento del ApS, en el que describían la experiencia de ApS que conocían.

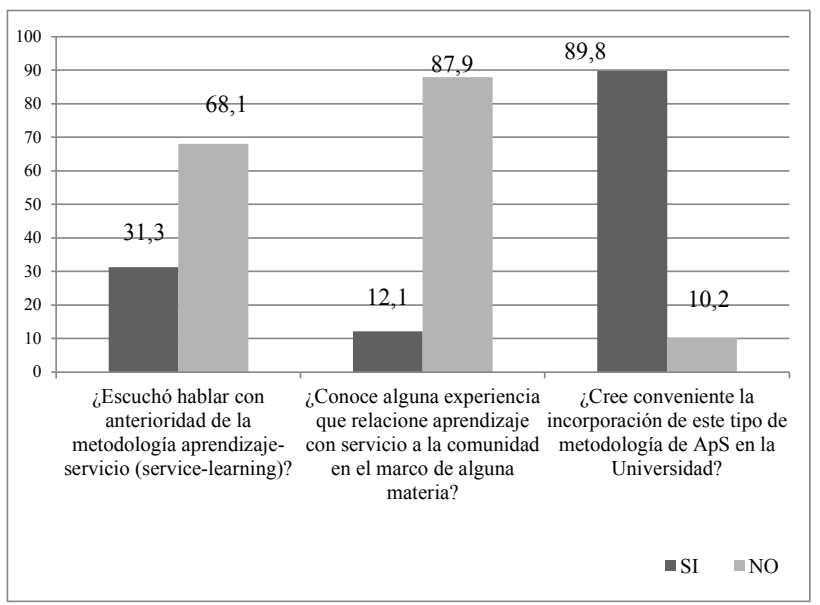

Gráfico 2: Conocimiento y pertinencia del aprendizaje-servicio

Para finalizar, las últimas cuestiones se refieren al conocimiento del aprendizaje-servicio. Los resultados obtenidos a partir de la prueba de chi-cuadrado nos indican que no existen diferencias significativas en las respuestas de los grupos de profesores. Aun así, nos gustaría comentar varios aspectos. En primer lugar, no 
resulta nuevo afirmar que los profesores que pertenecen a entidades cívico-sociales obtienen resultados más positivos que sus colegas, ya que son más los que conocen $(32,7 \%)$ el aprendizaje-servicio $(27,3 \%$ en el otro grupo), y experiencias $(15,1 \%)$ que relacionan aprendizaje y servicio a la comunidad $(8,3 \%$ en el otro grupo). En todo caso, ambos grupos ven positiva la incorporación del $\mathrm{ApS}$ a la universidad, aunque, curiosamente, ahora son los profesores que dicen no pertenecer a ninguna asociación los que más lo defienden $(91,1 \%)$.

\section{Discusión}

En definitiva, hemos podido observar como los profesores que pertenecen a asociaciones y entidades de corte cívico se distancian de sus compañeros a la hora de desarrollar su actividad docente (invitan más a profesionales externos, sus alumnos participan activamente en el aula, recomiendan a los alumnos visitar exposiciones o asistir a actos, promueven y organizan actividades complementarias, hacen evaluación continua y valoran la implicación de los alumnos y promueven actividades que fomentan el pensamiento crítico) y en la manera de entender la relación entre universidad y sociedad por medio de la responsabilidad social. Donde no se aprecian diferencias es en el conocimiento y pertinencia del aprendizaje-servicio, ya que en ambos grupos las respuestas son similares.

Lo que se extrae de los resultados es que la implicación en proyectos vinculados a estas entidades, parece que se relaciona con una mirada de la docencia más allá de las aulas, así como a entender la responsabilidad social de la Universidad como un factor clave para su desarrollo.

\section{Referencias}

Arias, S. \& Simón, A. (2004). Las Estructuras Solidarias de las Universidades Españolas: Organización y Funcionamiento. Madrid: Universidad Autónoma de Madrid - Fundación Telefónica.

Bowen, H. (1984). Graduate education and social responsibility. New Directions for Higher Education, 46, 113-119.

Consello Social da Universidade de Santiago de Compostela (2013). 25 anos do Consello social da Universidade de Santiago. Santiago de Compostela. Santiago de Compostela: Servizo de Publicacións e Intercambio Científico da USC.

Del Rincón, D., Arnal, J., Latorre, A. y Sans, A. (1995). Técnicas de investigación en ciencias sociales. Madrid: Dykinson.

Gaete, R. (2011). La responsabilidad social universitaria como desafío para la gestión estratégica de la Educación Superior: el caso de España. Revista de Educación, 355, 109-133.

García, R., Escámez, J., Martínez, M. \& Martínez, M.J. (2008). Aprendizaje de ciudadanía y educación superior. En S. Valdivieso, S. \& A. Almeida (Eds.), Educación y ciudadanía, Actas del XXVII Seminario Interuniversitario de Teoría de la Educación (pp. 81-120). Las Palmas de Gran Canaria: SITE.
Kymlicka, W. (2003). La política vernácula. Nacionalismo, multiculturalismo y ciudadanía. Barcelona: Paidós.

Lorenzo, M. \& Santos Rego, M.A. (2009). Educación para a cidadanía e os profesores. Visión e desafío. Vigo: Edicións Xerais.

Lorenzo, M. (2012). La función social de la universidad y la formación del profesorado. Edetania: estudios y propuestas socio-educativas, 42, 25-38.

Martínez, M. (Ed.) (2008). Aprendizaje servicio y responsabilidad social de las universidades. Barcelona: Octaedro.

Otero, O.F. (1974). La participación en los centros educativos. Pamplona: Eunsa.

Puig, J.M. et al. (2009). Aprendizaje Servicio (ApS). Educación y compromiso cívico. Barcelona: Graó.

Santos Rego, M.A. \& Lorenzo, M. (2007). Universidade e construcción da sociedade civil. Vigo: Edicións Xerais.

Santos Rego, M.A. (2013). ¿Para cuándo las universidades en la agenda de una democracia fuerte? educación, aprendizaje y compromiso cívico en Norteamérica. Revista de Educación, 361, 565-590.

Santos Rego, M.A., Sotelino, A. \& Lorenzo, M. (2015). Aprendizaje-servicio y misión cívica de la Universidad. Una propuesta de desarrollo. Barcelona: Octaedro.

Sotelino, A. (2014). Aprendizaje-servicio en las universidades gallegas Evaluación y propuesta de desarrollo. (Tesis doctoral). Universidade de Santiago de Compostela, Santiago de Compostela.

Ugarte, C. \& Naval, C. (2010). Desarrollo de competencias profesionales en la educación superior. Un caso docente concreto. Revista electronica de Investigación Educativa, 12. 1-14. Recuperado de redie.uabc.mx/index.php/redie/article/view/251/412 